\title{
Determination of the relativistic corrections to the static inter-quark potential from lattice QCD
}

\author{
Miho Koma ${ }^{* \dagger}$ \\ Numazu College of Technology, Numazu 410-8501, Japan \\ E-mail: m-koma@numazu-ct.ac.jp \\ Yoshiaki Koma \\ Numazu College of Technology, Numazu 410-8501, Japan \\ E-mail: koma@numazu-ct.ac.jp

\section{Hartmut Wittig} \\ Institut für Kernphysik, Johannes Gutenberg-Universität Mainz, D-55099 Mainz, Germany \\ E-mail: wittigekph.uni-mainz.de
}

\begin{abstract}
We investigate the relativistic corrections to the static potential, i.e. the $O(1 / \mathrm{m})$ potential and the $O\left(1 / \mathrm{m}^{2}\right)$ spin-dependent potentials, in SU(3) lattice gauge theory. Utilizing the multilevel algorithm, we obtain a very clean signal for these potentials in the region $0.25 \mathrm{fm} \lesssim r \lesssim 1.2 \mathrm{fm}$. We confirm long-range nonperturbative contributions in the $O(1 / \mathrm{m})$ potential and $O\left(1 / \mathrm{m}^{2}\right)$ spinorbit potentials.
\end{abstract}

8th Conference Quark Confinement and the Hadron Spectrum

September 1-6, 2008

Mainz. Germany

\footnotetext{
* Speaker.

${ }^{\dagger}$ M.K. is supported by Japan Society for the Promotion of Science (JSPS), Grant-in-Aid for JSPS Fellows (20.40152). Y.K. is partially supported by the Ministry of Education, Science, Sports and Culture, Japan, Grant-inAid for Young Scientists (B) (2074149). The authors are also supported by JSPS and DFG under the Japan-Germany Research Cooperative Program. The main calculation has been performed on the NEC SX8 at Research Center for Nuclear Physics (RCNP), Osaka University, Japan.
} 


\section{Introduction}

A possible strategy of studying heavy quarkonium in QCD is to employ potential nonrelativistic QCD (pNRQCD) [1, 2], which is obtained by integrating out the scale above the heavy quark mass $m \gg \Lambda_{\mathrm{QCD}}$ and the scale $m v$, where $v$ is quark velocity. The effective hamiltonian of pNRQCD up to $O\left(1 / \mathrm{m}^{2}\right)[2]$ is then given by

$$
\begin{aligned}
H= & \frac{\vec{p}_{1}^{2}}{2 m_{1}}+\frac{\vec{p}_{2}^{2}}{2 m_{2}}+V^{(0)}(r)+\frac{1}{m_{1}} V^{(1,0)}(r)+\frac{1}{m_{2}} V^{(0,1)}(r) \\
& +\frac{1}{m_{1}^{2}} V^{(2,0)}(r)+\frac{1}{m_{2}^{2}} V^{(0,2)}(r)+\frac{1}{m_{1} m_{2}} V^{(1,1)}(r)+O\left(1 / m^{3}\right),
\end{aligned}
$$

where $m_{1}$ and $m_{2}$ denote the masses of quark and antiquark, placed at $\vec{r}_{1}$ and $\vec{r}_{2}$, respectively. The static inter-quark potential $V^{(0)}\left(r \equiv\left|\vec{r}_{1}-\vec{r}_{2}\right|\right)$ emerges, accompanied by relativistic corrections classified in powers of $1 / m$. The potentials $V^{(1,0)}(r)=V^{(0,1)}(r)\left(\equiv V^{(1)}(r)\right)$ are the corrections at $O(1 / m)$. The potentials $V^{(2,0)}(r), V^{(0,2)}(r)$, and $V^{(1,1)}(r)$ are the corrections at $O\left(1 / m^{2}\right)$, which contain the leading order velocity-dependent potentials [3, 4] and spin-dependent potentials [5, 6]. The spin-dependent potentials are conventionally parametrized as

$$
\begin{aligned}
V_{\mathrm{SD}}(r) & =\left(\frac{\vec{s}_{1} \cdot \vec{l}_{1}}{2 m_{1}^{2}}-\frac{\vec{s}_{2} \cdot \vec{l}_{2}}{2 m_{2}^{2}}\right)\left(\frac{V^{(0) \prime}(r)}{r}+2 \frac{V_{1}^{\prime}(r)}{r}\right)+\left(\frac{\vec{s}_{2} \cdot \vec{l}_{1}}{2 m_{1} m_{2}}-\frac{\vec{s}_{1} \cdot \vec{l}_{2}}{2 m_{1} m_{2}}\right) \frac{V_{2}^{\prime}(r)}{r} \\
& +\frac{1}{m_{1} m_{2}}\left(\frac{\left(\vec{s}_{1} \cdot \vec{r}\right)\left(\vec{s}_{2} \cdot \vec{r}\right)}{r^{2}}-\frac{\vec{s}_{1} \cdot \vec{s}_{2}}{3}\right) V_{3}(r)+\frac{\vec{s}_{1} \cdot \vec{s}_{2}}{3 m_{1} m_{2}} V_{4}(r),
\end{aligned}
$$

where $\vec{s}_{1}$ and $\vec{s}_{2}$ denote the spins, and $\vec{l}_{1}=-\vec{l}_{2}=\vec{l}$ the orbital angular momenta.

Once these potentials are determined from QCD, various properties of heavy quarkonium can be investigated systematically by solving the Schrödinger equation. Since the binding energy of a quark-antiquark system is typically of the scale $m v^{2}$, which can be of the same order as $\Lambda_{\mathrm{QCD}}$ due to the nonrelativistic nature of the system, $v \ll 1$, as well as the fact that perturbation theory cannot incorporate quark confinement, it is essential to determine the potentials nonperturbatively.

Monte Carlo simulations of lattice QCD offer a powerful tool for the nonperturbative determination of the potentials. Recently, we investigated the $O(1 / \mathrm{m})$ potential $[7]$, and the $O\left(1 / \mathrm{m}^{2}\right)$ spin-dependent [9, 10] and momentum-dependent potentials [8] on a lattice utilizing the multilevel algorithm [11], and obtained remarkably clean signals up to distances of around $0.6 \mathrm{fm}$. In certaincases we observed deviations from the perturbative potentials.

In this report we present updated results of the $O(1 / \mathrm{m})$ potential and $O\left(1 / \mathrm{m}^{2}\right)$ spin-dependent potentials. In particular, we aim to clarify the long-distance behavior of these corrections.

\section{Formulation and Numerical Procedures}

According to pNRQCD, the $O(1 / \mathrm{m})$ and $O\left(1 / \mathrm{m}^{2}\right)$ potentials can generally be expressed by the matrix elements and the energy gaps which appear in the spectral representation of the colorelectric and color-magnetic field strength correlators (FSCs) on the quark-antiquark source [1], where we adopt the Polyakov loop correlation function (PLCF) as a quark-antiquark source. By utilizing the multilevel algorithm [11], we measure these FSCs of various geometries on a $V=L^{3} T$ 
Table 1: Simulation parameters used in this study. $N_{\text {tsl }}$ is the number of time slices in a sublattice and $N_{\text {iupd }}$ the number of internal update within a sublattice, both are parameters for the multilevel algorithm. The lattice spacing $a$ is set from the Sommer scale $r_{0}=0.5 \mathrm{fm}$.

\begin{tabular}{ccc|ccc|ccc}
\hline \multirow{2}{*}{$\beta=6 / g^{2}$} & \multirow{2}{*}{$a[\mathrm{fm}]$} & \multirow{2}{*}{$N_{\text {tsl }}$} & \multicolumn{3}{|c|}{ Spin-dependent potential } & \multicolumn{4}{|c}{$O(1 / m)$ potential } \\
& & & $(L / a)^{3}(T / a)$ & $N_{\text {iupd }}$ & $N_{\text {conf }}$ & $(L / a)^{3}(T / a)$ & $N_{\text {iupd }}$ & $N_{\text {conf }}$ \\
\hline 5.85 & 0.123 & 3 & $24^{4}$ & 50000 & 77 & $24^{4}$ & 50000 & 124 \\
6.00 & 0.093 & 4 & $20^{3} 40$ & 7000 & 33 & $24^{3} 32$ & 50000 & 100 \\
6.20 & 0.063 & 5 & & - & & $30^{3} 40$ & 50000 & 39 \\
6.30 & 0.059 & 6 & $24^{4}$ & 6000 & 39 & \multicolumn{2}{c}{-} & \\
\hline
\end{tabular}

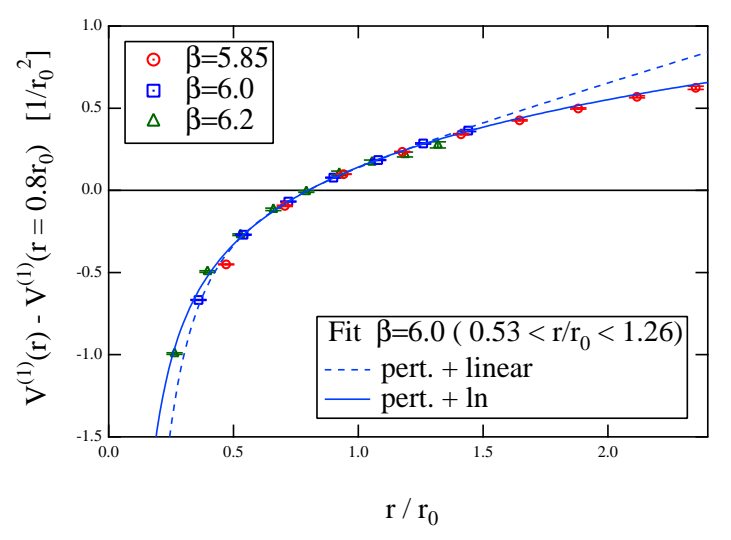

Figure 1: The $O(1 / m)$ potential $V^{(1)}(r)$. Two lines are the fit results obtained from the data at $\beta=6.0$ and $0.53<r / r_{0}<1.26$ (5 points) with the two fit functions, $V_{\text {linear }}(r)=-A_{1} / r^{2}+B_{1} r+C_{1}$ and $V_{\ln }(r)=-A_{2} / r^{2}+B_{2} \ln r+C_{2}$. Both functional forms can describe the data behavior within the fit window, however, $V_{\ln }(r)$ seems to be favored for the long-range data around $r \sim 2.0 r_{0}$.

lattice with lattice spacing $a$. Exploiting the spectral representation, we can extract the matrix elements and the energy gaps from the measured FSCs. Definitions and all technical details are described in Refs. [77, 8, 10].

\section{Numerical results}

We carry out simulations using the standard Wilson gauge action in SU(3) lattice gauge theory. We summarize our simulation parameters in Table1. In Fig. 2] we show the $O(1 / m)$ potential, $V^{(1)}(r)$, normalized at $r=0.8 r_{0}$ together with the two fit curves $V_{\text {linear }}(r)=-A_{1} / r^{2}+B_{1} r+C_{1}$ and $V_{\ln }(r)=-A_{2} / r^{2}+B_{2} \ln r+C_{2}$. Perturbation theory at $O\left(\alpha_{s}^{2}\right)$ provides $V_{\text {pert }}(r)=-\frac{C_{F} C_{A} \alpha_{s}^{2}}{4 r^{2}}$, where $C_{F}$ and $C_{A}$ are the Casimir charges of the fundamental and the adjoint representations, respectively, and $\alpha_{s}=g^{2} /(4 \pi)$ the strong coupling [1]. Owing to using larger spatial volumes compared to [7] and a re-tuning of the algorithm, we have now succeeded in determining $V^{(1)}(r)$ up to $r=2.35 r_{0} \sim 1.2 \mathrm{fm}$. Results for $V^{(1)}(r)$ at different $\beta$ show a reasonable scaling behavior, except for the data at $r / a=2$, which are likely to suffer from discretization errors. We confirm that the $O(1 / m)$ potential contains nonperturbative contribution. The new data set at $r \gtrsim 1.5 r_{0}$ suggest that the nonperturbative contribution may be described by a logarithmic function, as proposed in [12]. It is quite interesting to examine the effect of the $O(1 / \mathrm{m})$ potential on the spectrum as this is the leading-order relativistic correction in the $1 / m$ expansion. 

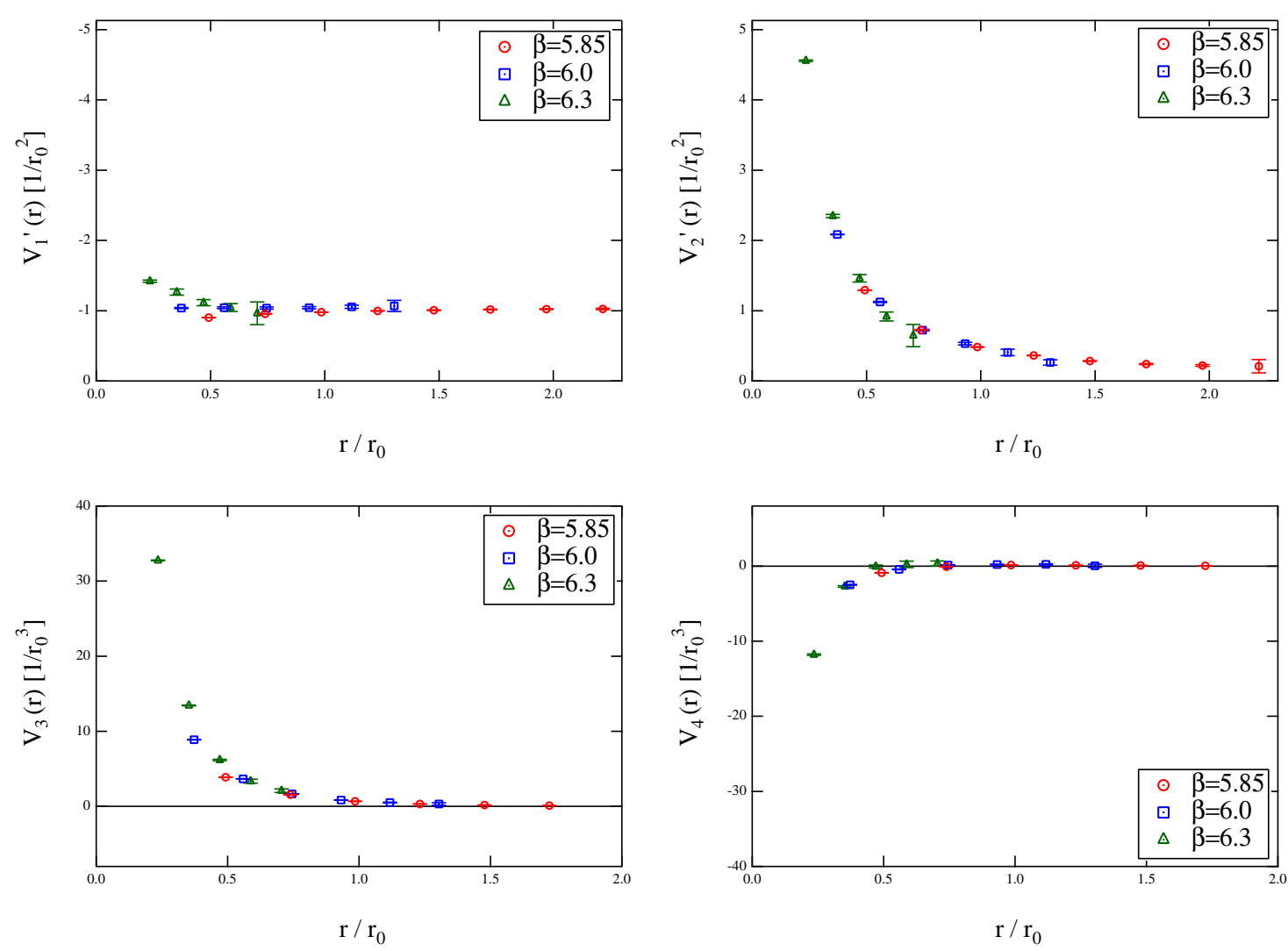

Figure 2: The spin-dependent potentials $V_{1}^{\prime}(r), V_{2}^{\prime}(r), V_{3}(r)$, and $V_{4}(r)$.

In Fig. 2. we present the spin-dependent potentials, $V_{1}^{\prime}(r), V_{2}^{\prime}(r), V_{3}(r)$, and $V_{4}(r)^{1}$. The $O\left(1 / m^{2}\right)$ spin-orbit potentials, $V_{1}^{\prime}(r)$ and $V_{2}^{\prime}(r)$, are non-vanishing up to $r=2.23 r_{0}$. The finite tail of $V_{2}^{\prime}(r)$ is an example of the observed deviation from perturbative potentials [10]. The Gromes relation, which is an important analytic relation derived from the Lorentz invariance, $V^{(0) \prime}(r)=V_{2}^{\prime}(r)-V_{1}^{\prime}(r)$ [6], is approximately satisfied as shown in Fig. 3 where the deviation from the relation $1-\left(V_{2}^{\prime}-V_{1}^{\prime}\right) / V^{(0) \prime}$ is plotted. The deviation is 10 to $12 \%$ at $a=0.123 \mathrm{fm}$, while 4 to $10 \%$ at $a=0.093 \mathrm{fm}$. It will be interesting to study whether the deviation vanishes after taking the continuum limit. For the spinspin potentials, $V_{3}(r)$ and $V_{4}(r)$, we confirm that they have no long-range contribution. In order to investigate their functional form, we definitely need data at $r \lesssim 0.5 r_{0}$.

\section{Summary}

We have investigated the relativistic corrections to the static potential, the $O(1 / m)$ potential and the $O\left(1 / \mathrm{m}^{2}\right)$ spin-dependent potentials, in SU(3) lattice gauge theory. They are important ingredients of the pNRQCD hamiltonian for heavy quarkonium. By evaluating the color-electric and color-magnetic FSCs on the PLCF with the multilevel algorithm, and exploiting the spectral

\footnotetext{
${ }^{1}$ The data at $\beta=6.0$ and 6.3 are already published in Ref. [10].
} 


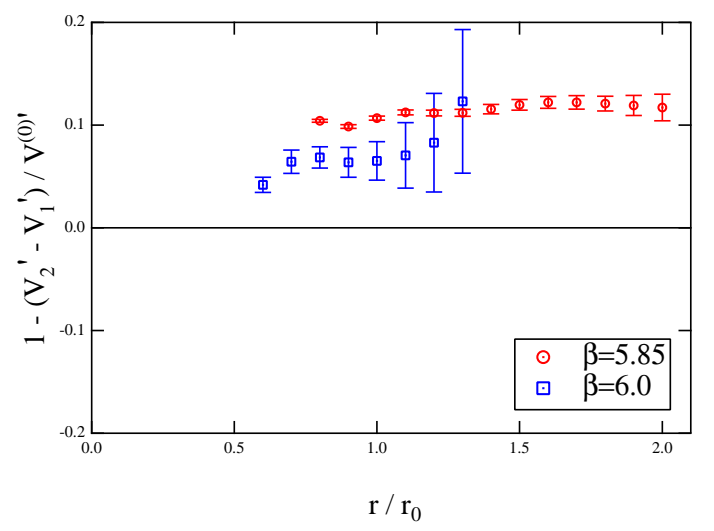

Figure 3: The quantity $1-\left(V_{2}^{\prime}-V_{1}^{\prime}\right) / V^{(0) \prime}$ is plotted as a function of $r$. If the Gromes relation is exactly satisfied, this quantity should be zero at all $r$.

representation of the correlator, we have obtained a very clean signal for these potentials in the region $0.5 r_{0} \lesssim r \lesssim 2.4 r_{0}$.

\section{References}

[1] N. Brambilla, A. Pineda, J. Soto, and A. Vairo, The qcd potential at $O(1 / m)$, Phys. Rev. D63 (2001) 014023, hep-ph/0002250.

[2] A. Pineda and A. Vairo, The QCD potential at $O\left(1 / \mathrm{m}^{2}\right)$ : Complete spin-dependent and spin-independent result, Phys. Rev. D63 (2001) 054007, hep-ph/0009145, Erratum-ibid D64, 039902 (2001)].

[3] A. Barchielli, E. Montaldi, and G. M. Prosperi, On a systematic derivation of the quark-antiquark potential, Nucl. Phys. B296 (1988) 625, Erratum-ibid B303, 752 (1988).

[4] A. Barchielli, N. Brambilla, and G. M. Prosperi, Relativistic corrections to the quark-antiquark potential and the quarkonium spectrum, Nuovo Cim. A103 (1990) 59.

[5] E. Eichten and F. Feinberg, Spin dependent forces in heavy quark systems, Phys. Rev. Lett. 43 (1979) 1205.

[6] D. Gromes, Relativistic corrections to the long range quark anti-quark potential, electric flux tubes, and area law, Z. Phys. C22 (1984) 265.

[7] Y. Koma, M. Koma, and H. Wittig, Nonperturbative determination of the QCD potential at $O(1 / \mathrm{m})$, Phys. Rev. Lett. 97 (2006) 122003, hep-lat/0607009.

[8] Y. Koma, M. Koma, and H. Wittig, Relativistic corrections to the static potential at $O(1 / \mathrm{m})$ and $O\left(1 / m^{2}\right), \operatorname{PoS}$ LAT2007 (2007) 111, 0711.2322.

[9] M. Koma, Y. Koma, and H. Wittig, Determination of the spin-dependent potentials with the multi-level algorithm, PoS LAT2005 (2005) 216, hep-lat/0510059.

[10] Y. Koma and M. Koma, Spin-dependent potentials from lattice QCD, Nucl. Phys. B769 (2007) 79-107, hep-lat/0609078.

[11] M. Lüscher and P. Weisz, Locality and exponential error reduction in numerical lattice gauge theory, JHEP 09 (2001) 010, hep-lat/0108014.

[12] G. Perez-Nadal and J. Soto, Effective string theory constraints on the long distance behavior of the subleading potentials, 0811.2762 